\title{
Strategy Planning for Collaborative Humanoid Soccer Robots based on Principle Solution
}

\author{
Cheng Yee Low, Norheliena Aziz \\ Centre of Excellence for Humanoid Robots and Bio-Sensing, Universiti Teknologi MARA, \\ Malaysia \\ e-mail: chengyee.low@salam.uitm.edu.my
}

\author{
Mustafa Aldemir \\ Siemens AS, Corporate Technology Development Center Istanbul, Turkey
}

\begin{abstract}
Martin Mellado
Instituto de Automática e Informática Industrial, Universtat Politècnica de València, Spain
\end{abstract}

Roman Dumitrescu, Harald Anacker

Project Group Mechatronic Systems Design, Fraunhofer Institute for Production Technology, Germany

\begin{abstract}
Collaborative humanoid soccer robots are currently under the lime light in the rapidly advancing research area of multi-robot systems. With new functionalities of software and hardware, they are becoming more versatile, robust and agile in response to the changes in the environment under dynamic conditions. This work focuses on a new approach for strategy planning of humanoid soccer robot teams as in the RoboCup Standard Platform League. The key element of the approach is a holistic system model of the principle solution encompassing various strategies of a soccer robot team. The benefits of the modelbased approach are twofold - it enables intuitive behavioral specification of the humanoid soccer robots in line with the team strategies envisaged by the system developers, and it systematicizes the realization of their collaborative behaviors based on the principle solution. The principle solution is modeled with the newly developed specification technique CONSENS ${ }^{\circledR}$ for the conceptual design of mechatronic and selfoptimizing systems.
\end{abstract}

Keywords: humanoid robotics, mechatronics, principle solution, intelligent technical systems, design methodology 


\section{Introduction}

In the recent years, robots work in a group to carry out collaborative tasks rather than operating individually. Such multi-robot systems play a prominent role in shaping the future of robotics research as they can perform tasks that single-robot systems may have difficulties to accomplish. An example under the lime light is the humanoid robot soccer team as in the RoboCup Standard Platform League (SPL) [1]. The ultimate goal of the RoboCup initiative is a team of fully autonomous humanoid robot soccer players that shall win a soccer game, complying with the official FIFA rules, against the winner of the most recent World Cup of human soccer by 2050. Though it sounds ambitious, research has been accumulating promising results. On one hand, the hardware has evolved to allow fast and stable locomotion and precise sensing. On the other hand, the software has evolved to enable complex operations such object recognition, bipedal walking and partial intelligence.

In SPL, all teams must use an identical humanoid robot platform, which is the NAO produced by Aldebaran Robotics. Previously, emphasis was put on realizing the functions of an individual robot so that it can shoot, pass, dribble, localize and search for a ball. So far most of the teams still do not take the advantages of coordination between the players. The lack of coordination between the players jeopardizes the performance of a team as human-like tactics such as passing and keeping formation is impossible without coordination. In some cases, more than one player of a team try to capture the ball, blocking each others' path, and even pushing each other.

Due to the increasing team size, an effective strategy planning taking the advantages of coordination between players becomes the success factor for a team [2-5]. It is fascinating to think of how these robots can implement a team strategy collaboratively and autonomously, for instance, during formations for kick-off positioning or collectively putting pressure on one opponent. However, this would not be possible without a systematic model-based approach during the strategy planning phase of these humanoid robot soccer teams. Indeed, various design issues prevail during strategy planning such as those pertaining to task assignment, hierarchy and organization, reliability, deployment and formation control, and scalability of team size.

In this paper, we present an approach for strategy planning of collaborative humanoid soccer robots based on the specification of a principle solution. Section 2 introduces a hierarchical structure for a humanoid robot soccer team. The specification technique CONSENS ${ }^{\circledR}$ is described in Section 3. Subsequently Section 4 exemplifies the 
strategy planning of a humanoid robot soccer team using the aforementioned specification technique. Finally, Section 5 concludes the outcomes of this work.

\section{Structure of Humanoid Robot Soccer Team}

A humanoid robot soccer team can be described by means of a hierarchical structure suggested by Lückel [6] for complex mechatronic systems. The basis consists of so-called mechatronic function modules (MFM) that comprise a basic mechanical basic structure, sensors, actuators and a local information processing, which contains the controller. MFMs that are connected by information technology and/or mechanical elements result in autonomous mechatronic systems (AMS). They also feature information processing. Within this information processing, superior tasks are being realized, such as monitoring, fault diagnosis and maintenance decisions. Additionally targets for the local information processing of the MFM are being generated. AMS form the so-called networked mechatronic systems (NMS). NMS are produced just by connecting the AMSs via information processing. Similar to the AMS, the information processing of the NMS realizes superior tasks. Transferring these terms to the context of this work, a bipedal locomotion module would be considered to be a MFM, a NAO robot which consists of the bipedal locomotion module and the other MFMs would be considered an AMS, and a team of NAO robots would be considered a NMS. On every level within this structure, it is possible to complement the controllers by the functionality of self-optimization. By this, the regarded system's elements (MFM, AMS, NMS) gain inherent partial intelligence. The behavior of the whole system is formed by the communication and cooperation of the intelligent system elements. From an information processing point of view we consider these distributed systems to be multi-agent-systems. 


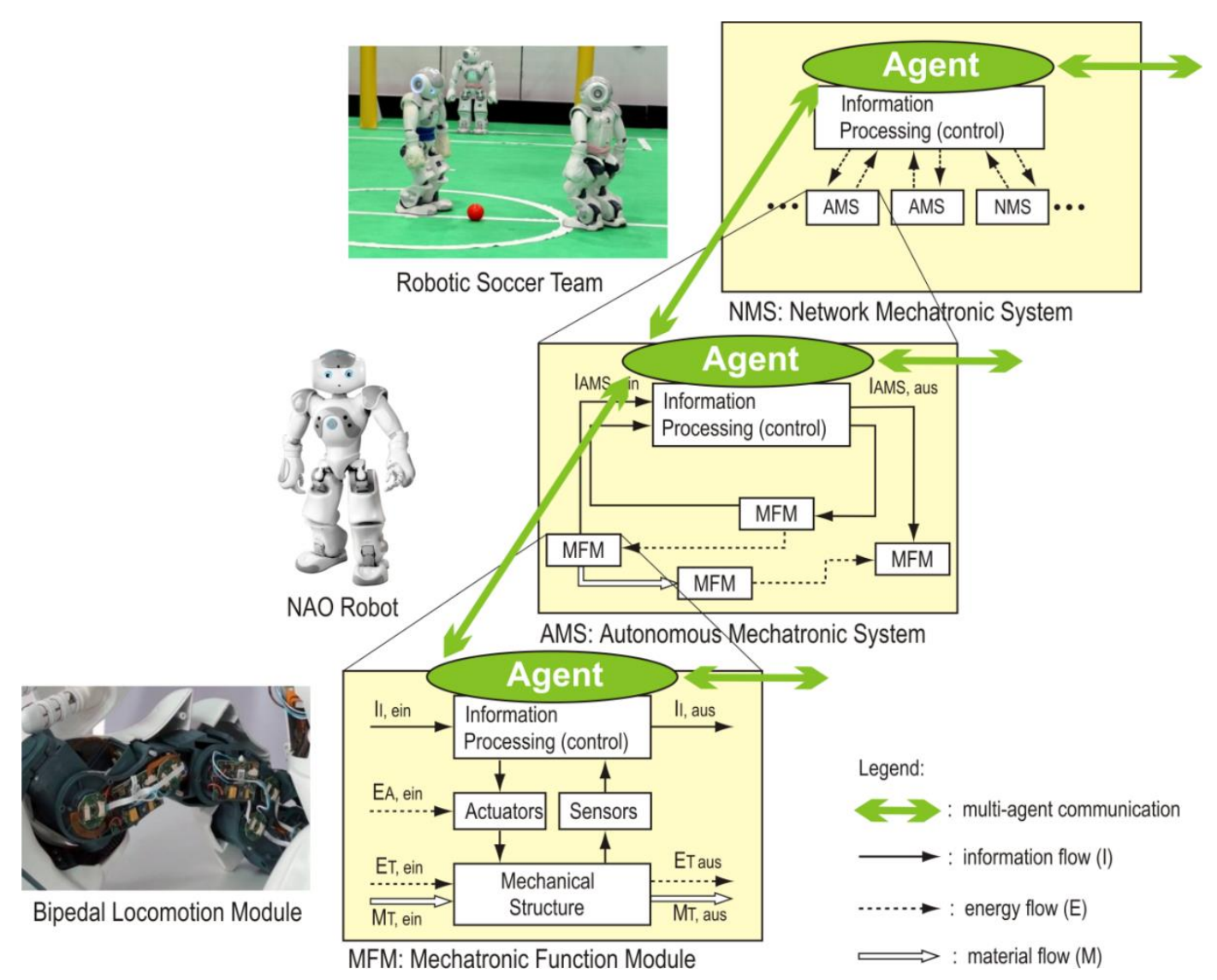

Figure 1. Hierarchical structure of humanoid robotic soccer team.

\section{Specification Technique}

During the system design of advanced mechatronic systems, a cross-domain system model is necessary, which combines all the essential aspects of mechanical, electrical and software engineering. This system model is the basis of the first analysis, verification and validation on the systems level and at the same time the initial point of specific concretization within the different domains [7]. To establish these requirements of model-based systems engineering, a specification technique called "CONceptual design Specification technique for the ENgineering of complex Systems" abbreviated as CONSENS $^{\circledR}$ has been developed to describe the principle solution of advanced mechatronic systems [8].

As shown in Figure 2, the following aspects need to be taken into account: requirements, environment, application scenarios, functions, active structure, system of objectives, shape and behavior. The aspect behavior consists of a whole group because there are different kinds of behavior, e.g. the logic behavior, the dynamic behavior of multi-body systems, the cooperative behavior of system components, etc. These aspects 
are computer intern represented by partial models. A software tool called the Mechatronic Modeller ${ }^{\circledR}$ can be used to describe mechatronic systems using the specification technique. The Mechatronic Modeller ${ }^{\circledR}$ offers a separate editor for each partial model. The partial models are intertwined and form a coherent system model. By using this specification technique, the system that is to be developed can be described in an integrated, domainspanning way.

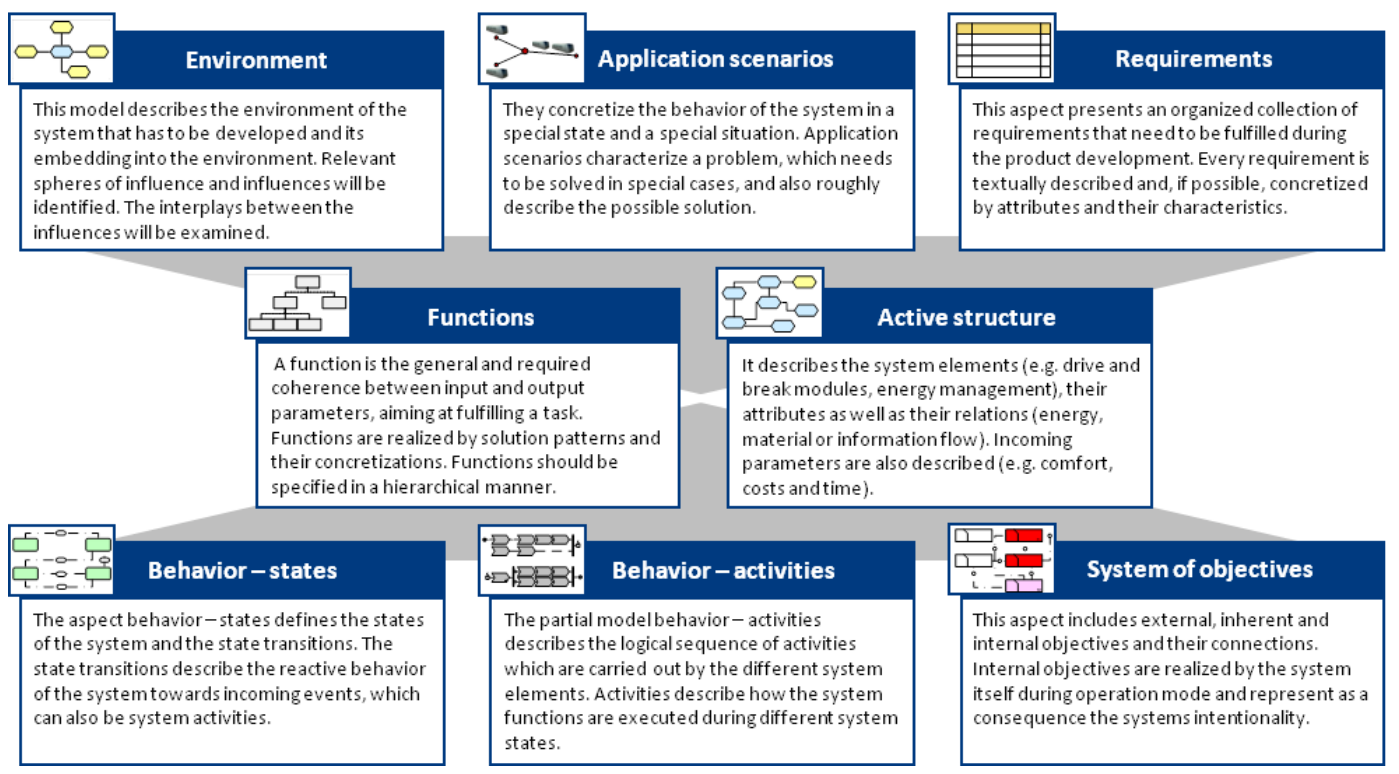

Figure 2. Partial models for the domain-spanning description of the principle solution of advanced mechatronic systems

\section{Strategy Planning based on Principle Solution}

To facilitate the strategy planning in SPL, the basic structure and the mode of operation for a humanoid robot soccer team has to be described. Such principle solution serves as a basis for systematic implementation of team strategies. It also improves technical communication and cooperation among the experts.

\subsection{Functions}

This aspect concerns the hierarchical subdivision of the functionality of a humanoid robot soccer team. A function is the general and required coherence between input and output parameters, aiming at fulfilling a task. For the setting up of function hierarchies, there is a catalogue with functions which is based on Birkhofer [9] and 
Langlotz [10]. Functions are realized by solution patterns and their concretizations. A subdivision into sub-functions takes place until useful solution patterns are found for the functions. Figure 3 illustrates a function hierarchy for a humanoid soccer robot in a team play. The functions are described as follows.

- To move: This function fulfils the locomotion task. These tasks require the ability of the robot to walk on two legs either forward or backward, to turn to the left or to the right, and to get up when it falls down.

- To localize: This function fulfils the task to determine the position and orientation of the robot and the ball in the game field.

- To kick: This function fulfils the task to shoot a ball towards a goal or to pass a ball to a teammate.

- To recognize: This function fulfils the task to perceive objects, particularly to distinguish between teammates and opponents, and to recognize the ball, the field lines and the goalposts.

- To communicate: This function fulfils the task to pass messages between a robot and its teammates as well as between a robot and a game controller (software referee).

- To generate trajectory: This function fulfils the task to determine a path of motion for a robot, not only for obstacle avoidance and minimum distance, but also to ensure that when the robot reaches the ball, it will be more or less aligned with the ball. 


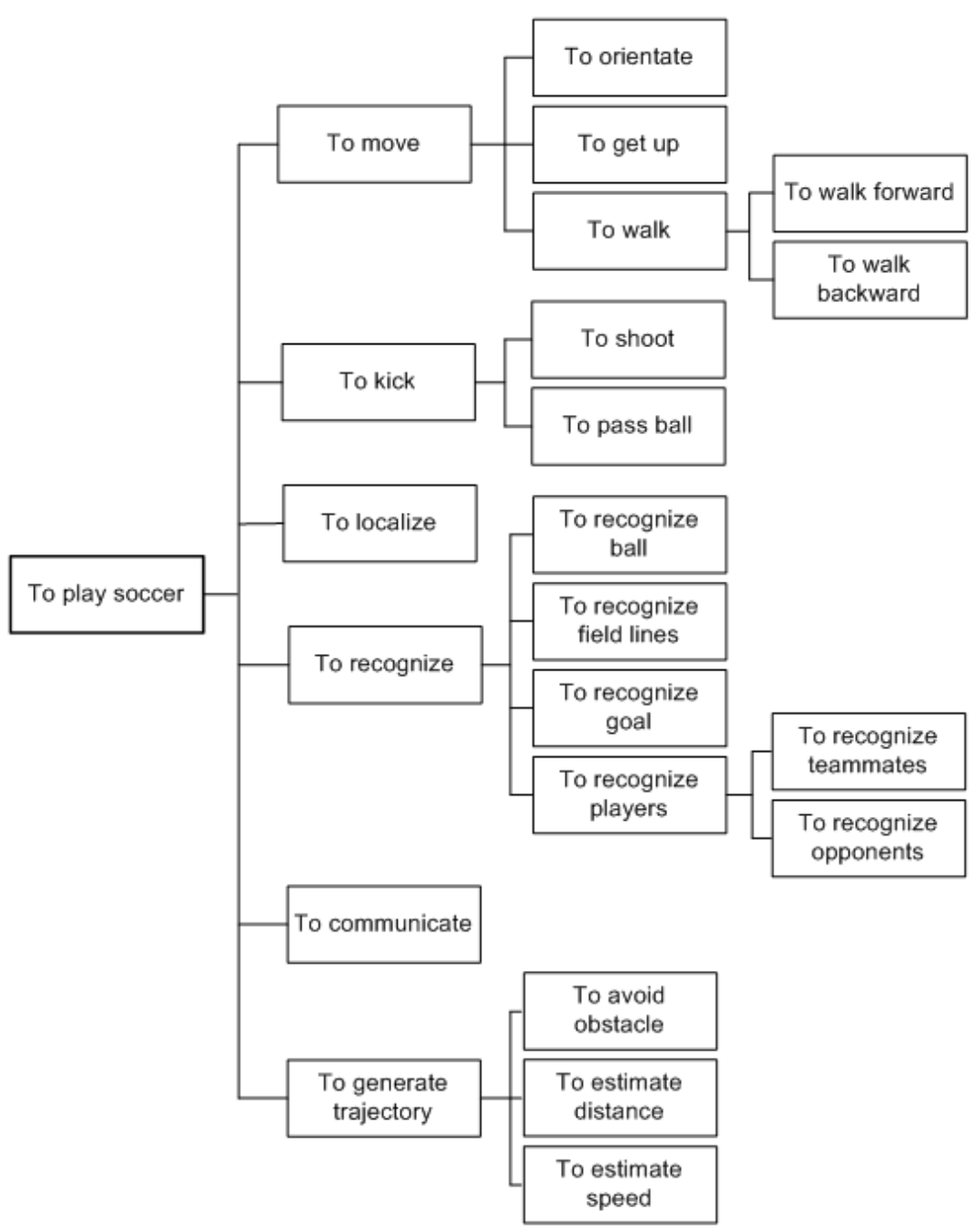

Figure 3. Functions of collaborative soccer robots

\subsection{Environment}

This model describes the environment of a humanoid soccer robot and its embedding into the environment. The relevant influences can be identified and the interplays between the influences can be examined. Figure 4 illustrates the environment of a soccer robot which consists of a ball, teammates, opponent players, goalposts, field lines and a game controller. The influence $I_{l}$ is exemplified in an influence table. The influences that trigger a state transition are marked as events, for instance, the visibility, possession and distance of the ball. Within such environment, the goal post sets the direction for a soccer robot, the opponent players are the obstacles to be avoided, the field lines define the boundaries of the game field, and the game controller transmits the commands from the referee to the soccer robot. 


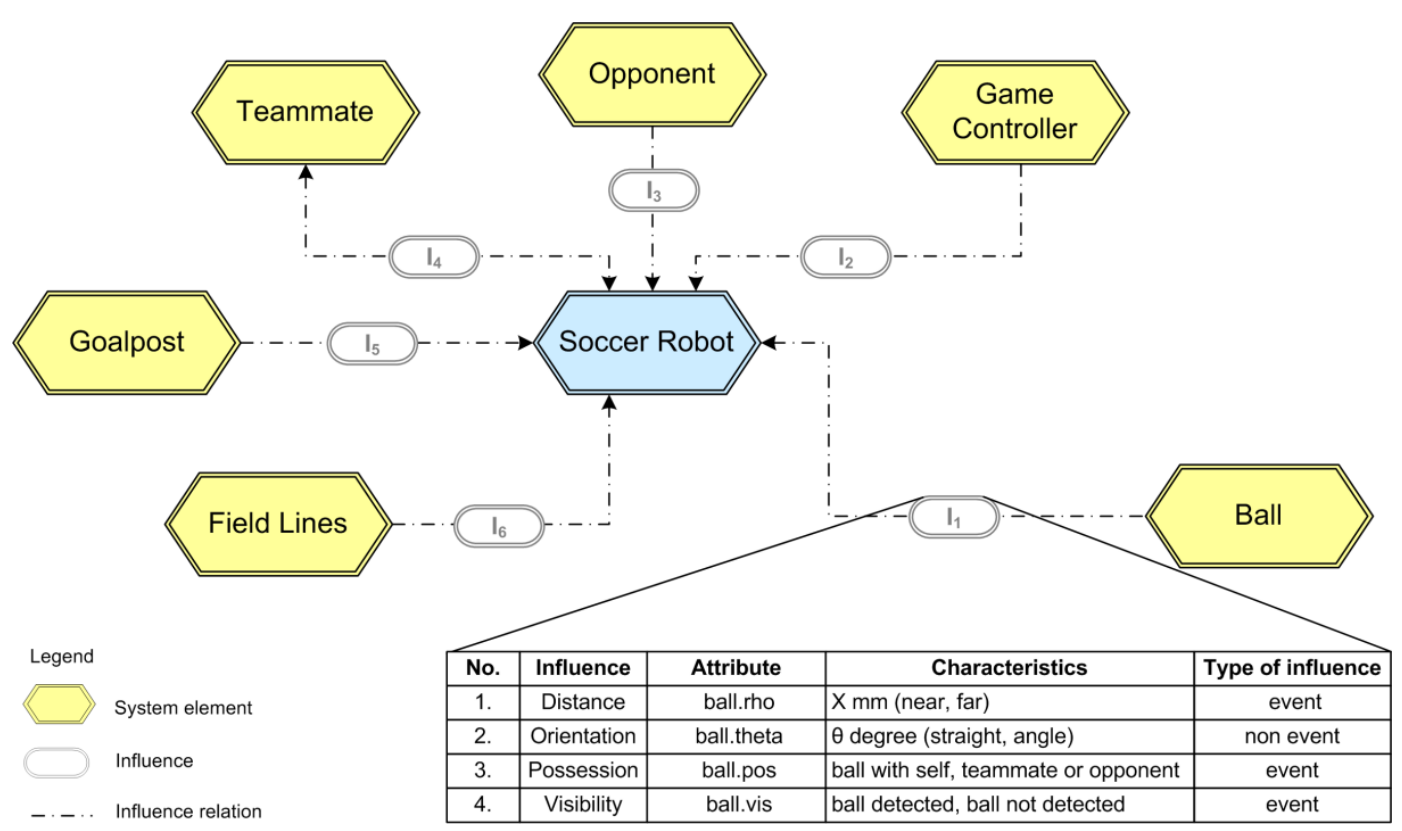

Figure 4. Environment model for a soccer robot in RoboCup

\subsection{Active Structure}

The active structure describes the system elements, their attributes as well as the relation of the system elements. It is the target to define the basic structure of the system, including all system configurations which can be thought ahead. Figure 5 shows a cut-out of an active structure for a soccer robot. Besides the hardware for the sensors and actuators, there are five software modules internal of a soccer robot, i.e. a Control Module, a Perception Anchoring Module, a Team Coordination Module, a Communication Module and a Commander Module. The software modules are described as follows. 


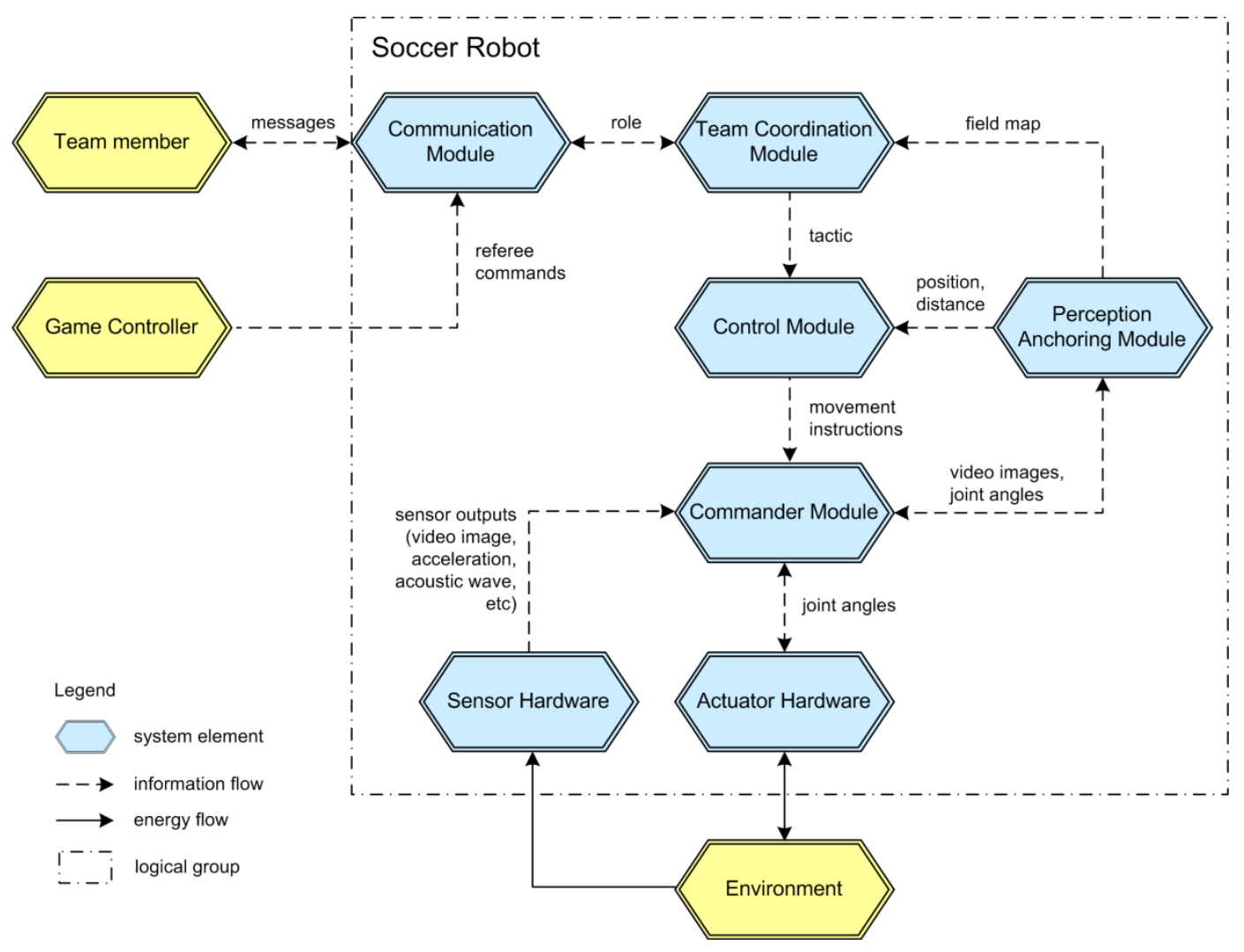

Figure 5. Active structure of a soccer robot

- The Communication Module enables a soccer robot to communicate with its team members. The inter-robot communication includes broadcasting to all robots and message passing between particular robots. Using the communication module, the robot also receives commands from the game controller which is software used by the referees to manage the game and broadcast messages about the game status to the robots. Both types of communication are based on User Datagram Protocol which is connectionless, involves no error checking and enables broadcasting and multicasting.

- The Team Coordination Module is responsible for tactical decision making concerning the strategy of team play in order to maximize the winning possibility. It makes decisions in terms of pre-defined behaviors with an aim to optimize the overall team performance. The outcome is the tactic pursued by a team (e.g. team attack, team defense or individual) and thus the role played by a soccer robot (e.g. striker, supporter or defender) for the current situation. The decisions made will be passed to the Control Module.

- The Control Module governs the behavior of a soccer robot by producing movement instructions with respect to the tactic determined by the Team 
Coordination Module. These instructions result in the activities of a robot, for instance, find the ball, go to ball, align with the ball, etc.

- The Commander Module receives movement instructions from the Control Module, interpreting them and executing them accordingly at the level of joint coordinates. It provides an interface for sensory-motor coupling integrating the actuators and the sensors. When a new order is received, it should be interpreted if it endangers the stability of the robot. It is not executed if it will cause instability.

- The Perception Anchoring Module maintains a consistent representation of the surrounding using the visual and odometry sensors in order to provide the necessary information to the Team Coordination Module, Control Module and Commander Module. It realizes functions such as image acquisition, colour based vision, object recognition, distance estimation and vision calibration.

These modules are highly interdependent and supporting each other during the game play. The Control Module can send a request to track a particular object. The Perception Anchoring Module will then track the position of the object using camera and sensors and send the information to the Control Module. Similarly, the Team Coordination Module also can send a tracking request to get the position information of the players and the ball. The Team Coordination Module will communicate with the other robots through the Communication Module and use another shared memory space to direct the Control Module to better decisions. The Control Module will use the information supplied to actuate the robot by giving orders to the Commander Module which implements them in actuators motions.

\subsection{Behavior}

The partial model behavior-state describes the envisaged system states, the state transitions, as well as the events that trigger a state transition. The partial model behavior-activity describes logical sequences of system activities which includes all operation and adaptation processes. Operation processes refer to the activities that are carried out within a state while adaptation processes refer to the activities that are carried out during state transitions. When an event appears, an adaptation process is triggered. 
After performing the adaptation process, the system takes over a new state and thus another set of operation processes are activated.

In the context of behavioral specification, the tactics used by a team (e.g. team attack, team defense, individual), the roles taken by each of the players (e.g. striker, supporter, defender, goalkeeper) and the activities to be implemented (e.g. find the ball, go to ball, align with the ball) must be clearly described, including their interrelations.

Figure 6 shows a cut-out of the partial model behavior-state for a humanoid soccer robot team. Each state in the figure corresponds to a tactic used by the team. At the highest level, there is a state that employs an individual tactic and another state that employs a team tactic. The state for team tactic consists of two sub-states, one employs a defense tactic while another employs an attack tactic. In the attacking state, a soccer robot can either be in the mode of a striker, passive defender or supporter. The activities to be executed in each of the states are also specified, as exemplified in Figure 7.

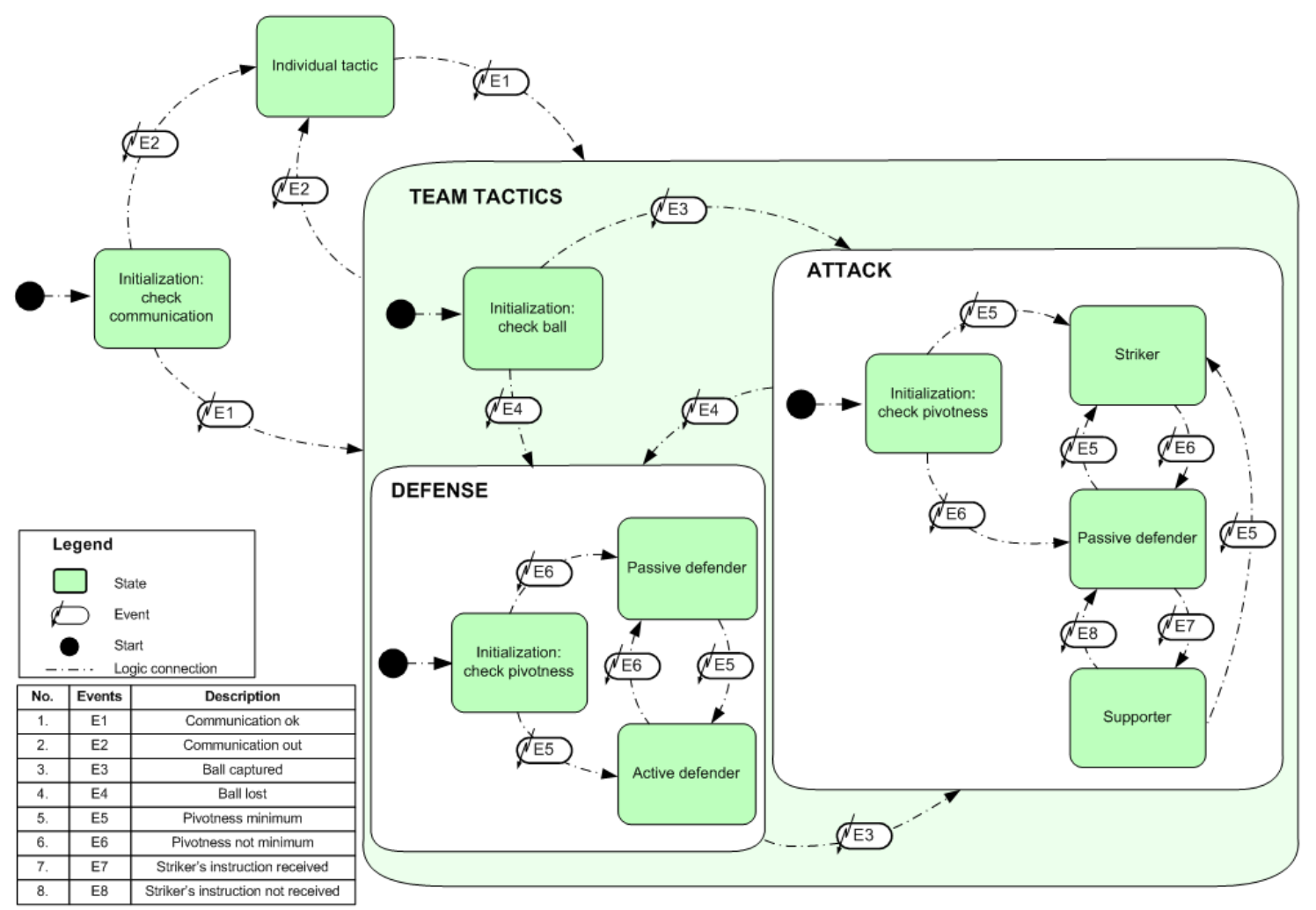

Figure 6. Hierarchical states of a soccer robot 

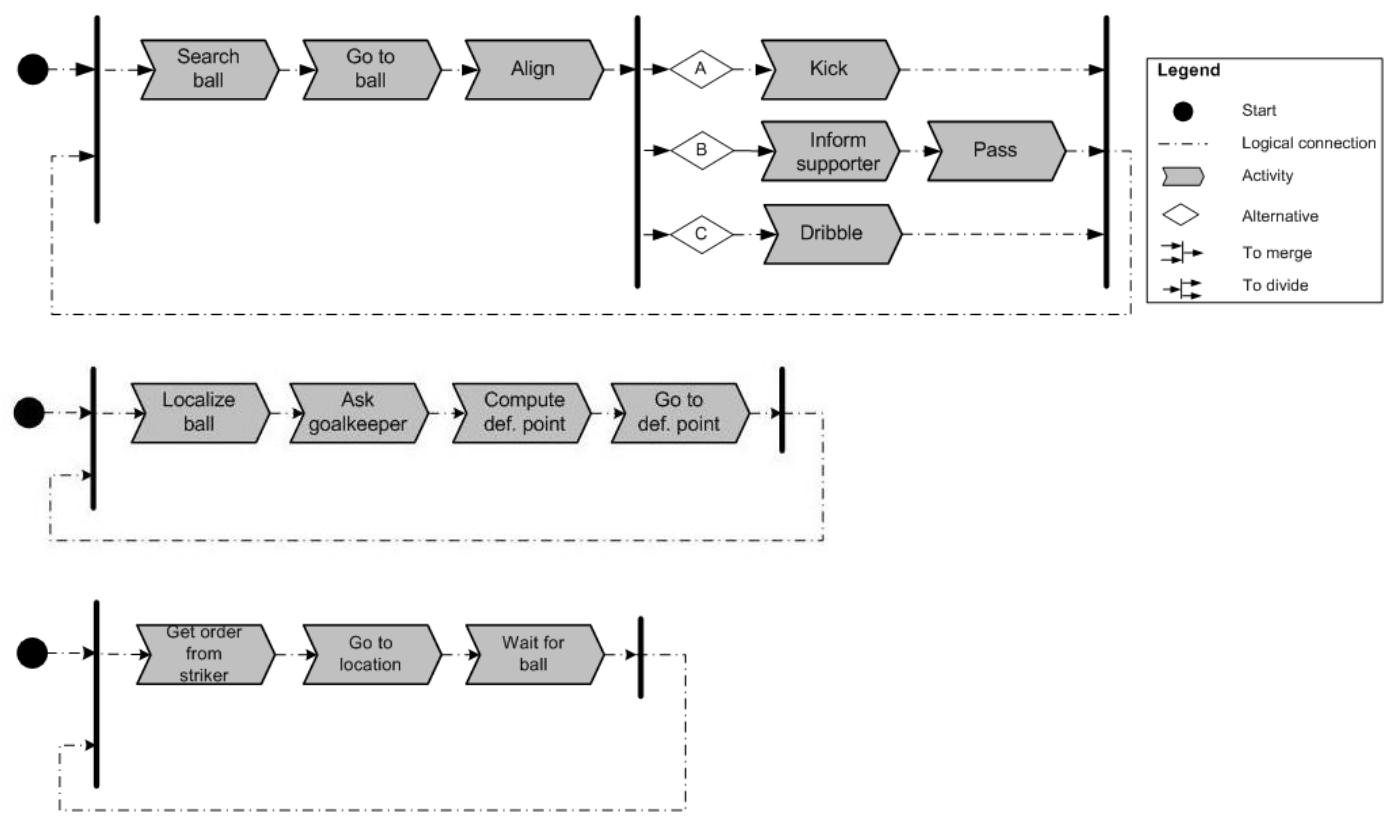

Figure 7. Activities executed by a striker (top), passive defender (middle) and supporter (bottom)

\section{A. Individual Tactic}

For a coordinated team play, network connection is required for communication among the players. Refer events $E 1$ and E2 in Figure 6. Thus, a player firstly checks its communication ability with the team mates. And if it cannot access its team mates, it plays completely individually until a network connection is available. Within the state "individual tactic", the player first searches the ball, and then goes to it. When it approaches close enough to the ball, it searches the opponent goal and aligns with the ball. Then, it shoots.

\section{B. Team Attack Tactic}

When a network connection is available, each player localizes itself on the field and estimates its distance to the ball, then sends this information to its teammates. This is referred as event E3. A state transition into "team attack tactic" takes place if any player of the team is close enough to the ball to kick it. If the visual recognition capability of the players is sufficient, the distance of the closest opponent can be taken into account.

Each player also calculates its cost (pivotness) to align with the ball for a good kick towards the opponent goal. Note that it may take a player at a shorter distance but opposite orientation longer than a player with a longer distance with a matching 
orientation. The player with the lowest cost transits into the state "striker". The striker role is exclusive; only one player can be in this role at a moment.

The main role of the striker is shooting. Its activities are shown in Figure 7. It goes to the ball, aligns with it and evaluates the feasibility of shooting. Three alternatives are possible. Feasibility for each alternative depends on the angle it sees the opponent goal open.

Alternative A: If the player is very far away from the opponent goal, or there is another player closing its sight; it's not feasible to shoot. If the feasibility is higher than a threshold value, it shoots.

Alternative B: If not, it orders the closest teammate (supporter) to go to a position where it can pass the ball and it kicks the ball with a reduced speed.

Alternative C: If no teammate is available or they are all too far away; the striker kicks the ball with a reduced speed and walks behind it to get a better position.

The player which is neither striker nor supporter takes the "passive defender" role. They communicate with the goalkeeper and get the best position to defend the goal from counter-shoots.

Each player localizes itself and sends this information to the other players periodically, and every time after a player kicks the ball. The roles can be interchanged if the cost value of striker is higher than another player. However, a hysteresis effect must be introduced to prevent fast role switching; the cost value required to lose the striker role is be higher than the cost value required to get the striker value.

\section{Team Defense Tactic}

If none of the players is close to the ball to kick it soon, refer event $E 4$, Team Defense Tactic is applied. The players localize themselves and calculate their cost values and share it with teammates. The player with the lowest cost gets the "active defender" role. This player goes towards the ball and tries to capture it while the other two players (passive defenders) try to close the sight of the goal in collaboration with the goalkeeper.

\section{Goalkeeper}

The task of the goalkeeper is taking the best position to close the sight of an opponent player. It localizes the ball (and the opponent player, if possible), and stand 
between the ball and the own goal. It also dictates the position of the passive defenders in order to minimize the angle the opponent can see the goal. If it detects that the ball is coming towards the goal fast, it can jump down or to the side to stop it.

\section{Conclusion}

The most obvious change in the rules of RoboCup SPL in the recent years was the increase of team size. It can be presumed that these rules will converge to the rules of a human football match. This emphasizes the importance of coordination. Thus a flexible approach for strategy planning is necessary in order to cope with the increased technical challenge. Following the approach presented in this paper, the strategy of the team play becomes very clear and intuitive. The approach systematizes the realization of the collaborative behavior of the humanoid soccer robots. The strategies defined in this paper will be adapted each year with new regulations, while the coordination of players will be constantly enhanced. If changes were required within the codes, the specific modifications can be recognized immediately by referring to the diagram without having to browse through the lengthy programming lines. Furthermore, contradictory specifications that lead to behavioral conflicts can be avoided and thus coordination can be enhanced.

\section{Acknowledgements}

This project is funded by the Ministry of Higher Education Malaysia under the grant number 600RMI/ST/FRGS 5/3/Fst (256/2010) and 600-RMI/ERGS 5/3 (23/2011). 


\section{References}

[1] Asada, M., Kitano, H., "The RoboCup Challenge" Robotics and Autonomous Systems 29 (1999) $3-12$

[2] Spaan, M. T. J., Groen, F. C. A., "Team Coordination through Roles, Positioning and Coordinated Procedures".

[3] Lau, N., Lopes, L. S., Corrente, G., Nelson,, F., "Multi-Robot Team Coordination through Roles, Positionings and Coordinated Procedures", 2009 IEEE/RSJ International Conference on Inte-lligent Robots and Systems, October 11-15, 2009 St. Louis, USA

[4] Iocchi, L., Nardi, D.,Piaggo, M., Sgorbissa, A., "Distributed Coordination in Heterogeneous MultiRobot Systems", Autonomous Robots 15, 155-168, 2003.

[5] Almeida, F., Lau, N., Reis, L. P., "A Survey on Coordination Methodologies for Simulated Robotic Soccer Teams".

[6] International Council on Systems Engineering (INCOSE): Systems Engineering Vision 2020. Incose-TP-2004-004-02, September 2007.

[7] Lückel. J., Hestermeyer, T., Liu-Henke, X., 2011. "Generalization of the Cascade Principle in View of Structured Form of Mechatronic Systems" IEEE/ASME International Conference on Advanced Intelligent Mechatronics ( AIM 2001), Villa Olmo, Como, Italy

[8] Gausemeier, J., Frank, U., Donoth, J., and Kahl, S., 2009, "Specification Technique for the Description of Self-Optimizing Mechatronic Systems," Research in Engineering Design, 20(4), pp. 201 223.

[9] Birkhofer, H., Analyse und Synthese der Funktionen Technischer Produkte. Dissertation, Technische Universität Braunschweig.

[10] Langlotz, G., 2000. Ein Beitrag zur Funktionsstrukturentwicklung Innovativer Produkte. Dissertation, Institut fu“r Rechneranwendung in Planung und Konstruktion, Universita"t Karlsruhe, ShakerVerlag, Band 2/2000,Aachen 